\title{
Infants' eyewitness testimony: Effects of postevent information on a prior memory representation
}

\author{
CAROLYN ROVEE-COLLIER, MARGARET A. BORZA, SCOTT A. ADLER, \\ and KIMBERLY BOLLER \\ Rutgers University, New Brunswick, New Jersey
}

\begin{abstract}
In eyewitness testimony research, postevent information impairs retention of the original event and increases the probability that interpolated information will be identified as part of the original event. The present experiments studied these effects with 3-month-olds. Infants learned to kick to move a particular crib mobile and then were briefly exposed to information about a novel mobile. The novel postevent information impaired recognition of the original mobile when it immediately followed training but not when it was delayed by 1 day. Like adults, infants treated the postevent information as part of the original training event, continuing to do so for at least 2 weeks. We propose that postevent information displaces conflicting information coactive with it in primary memory and creates a new, updated memory token of the event. Once the new token leaves primary memory, however, it is protected; only a copy can be retrieved and modified in the future.
\end{abstract}

It is well established that information that is encountered after an event can alter subsequent retention of that event. In animals, this problem has been studied largely in connection with the phenomenon of retroactive interference (Smith, 1968), consolidation (Lewis, 1979), or the administration of amnestic (Squire \& Davis, 1981) or memory-enhancing (McGaugh \& Dawson, 1971) agents. In humans, studies of retroactive interference date back more than a half century (Jenkins \& Dallenbach, 1924; McGeoch, 1942). Recent interest in this problem, however, was stimulated by E. F. Loftus's finding that college students' recollections of a crime or accident were modified by new information that was introduced after the event was witnessed (E. F. Loftus, 1975, 1979, 1981; E. F. Loftus, Miller, \& Burns, 1978).

Although research on adult eyewitness testimony has generated considerable controversy over the ultimate fate of the original memory representation (Bekerian \& Bowers, 1983; Belli, 1989; Christiaansen \& Ochalek, 1983; Lindsay, 1990; E. F. Loftus \& Hoffman, 1989; E. F. Loftus \& G. R. Loftus, 1980; E. F. Loftus, Schooler, \& Wagenaar, 1985; McCloskey \& Zaragoza, 1985; Tversky \& Tuchin, 1989; Zaragoza \& Koshmider, 1989; Zaragoza, McCloskey, \& Jamis, 1987), there is little disagreement that the postevent "misinformation effect" is real. Whether postevent information affects children's memories, however, is still controversial. Although

This research was supported by Grants MH32307 and MH00902 to C.R.-C. from the National Institute of Mental Health. We are grateful to Robert Belli for helpful suggestions and criticisms of earlier versions of this manuscript. Requests for reprints should be sent to C. RoveeCollier, Department of Psychology, Busch Campus, Rutgers University, New Brunswick, NJ 08903. recent evidence suggests that children, like adults, are susceptible to the misinformation effect (Goodman \& Aman, 1990; for review, see Ceci, Toglia, \& Ross, 1987, and Doris, 1991), this conclusion has been challenged: "The available evidence provides little support for the hypothesis that young children are susceptible to memory impairment. Without additional evidence in support of the memory impairment claim, the reliability and generality of this effect remains open to question" (Zaragoza, 1991, p. 36).

In studies with 3-month-olds, infants learned to kick to activate mobiles composed of blocks that displayed the same alphanumeric character (A or 2) on all sides (Greco, Hayne, \& Rovee-Collier, 1990; Hayne, Rovee-Collier, \& Perris, 1987). Each day, the characters on the mobiles were the same, but their color was different. During testing, infants transferred responding to a mobile displaying the training character in a novel color (e.g., a purple A) but did not respond to a mobile displaying identically colored characters from the other category (e.g., a purple 2). When infants were passively exposed to a highly physically dissimilar novel object that was functioning in the same manner as the category exemplars immediately after training was over, however, they subsequently transferred responding to that novel object as well as to other novel objects that they had not previously seen; if they were exposed to the same novel object functioning differently, they treated the test object as unique and failed to perform the learned response (Greco et al., 1990). Because infants were only passively exposed to the shared functional information, and because this information was physically absent during testing $24 \mathrm{~h}$ later, transfer to the highly physically dissimilar object could not have been achieved unless the object's attributes had somehow been 
integrated with the infants' memory of category training at the time it was exposed.

In the preceding studies, our focus was on the information that infants used to classify a novel object as a category member. Therefore, we did not assess the effect of introducing information about the novel object on the infant's memory of their prior training exemplars. The procedure by which information about the novel object was introduced, however, was virtually identical to the postevent-information procedure followed in studies of eyewitness testimony with children and adults (Ceci, Toglia, \& Ross, 1987; E. F. Loftus, 1975, 1979, 1982; E. F. Loftus et al., 1978). This commonality raised the possibility that the passive-exposure procedure could be adapted to study the effects of conflicting postevent information on infant memory within the framework of traditional research on eyewitness testimony. The succeeding experiments were designed to explore this possibility.

\section{EXPERIMENT 1}

Before proceeding directly to study the effects of postevent information on retention, we thought it prudent to ensure that the novel mobiles to be used during the postevent-information procedure were discriminably different to 3-month-olds. Although some of these mobile models had been used in previous discrimination studies with infants of this age, some had not.

In addition, Greco et al. (1990) had trained infants with a different mobile in each session (variable training) prior to introducing information about the novel object; however, in the present studies, we wanted to train infants with the same object in every session (constant training). Before proceeding with the postevent-information procedure, therefore, we thought it also important to compare the effects of variable and constant training on infants' 24-h test performance to novel and familiar mobiles. To this end, we trained independent groups of infants with either the same or a different mobile in each session and assessed their retention $24 \mathrm{~h}$ later either with the original (Session 1) mobile or with a completely novel one.

\section{Method}

Subjects. Twenty-four healthy and apparently normal infants (10 males, 14 females; $M$ age $=86.0$ days, $S D=6.9$ ) were recruited from the New Brunswick, $\mathrm{NJ}$, area through birth announcements in local newspapers and by word of mouth. Additional subjects were excluded as a result of crying for longer than 2 consecutive minutes ( $n=8$ ), failing to meet the learning criterion $(n=1)$, or a scheduling conflict $(n=2)$. The subjects were randomly assigned to one of four groups $(n=6)$, as they became available for study.

Apparatus. Reinforcement was provided by one of three handpainted wooden mobiles, identical in basic construction, size, complexity, and number of components but differing in theme, component shape, and prominent colors (Nursery Plastics, Inc., Models 801, 805, and 809; see Figure 1). Prior to participation, no infant had been exposed to any of these models, which are not commercially available. The models used during training and testing were counterbalanced within and across groups.

An inverted L-shaped, metal mobile stand (BCS, So. Plainfield, NJ) was clamped to opposite sides of the crib such that the over-
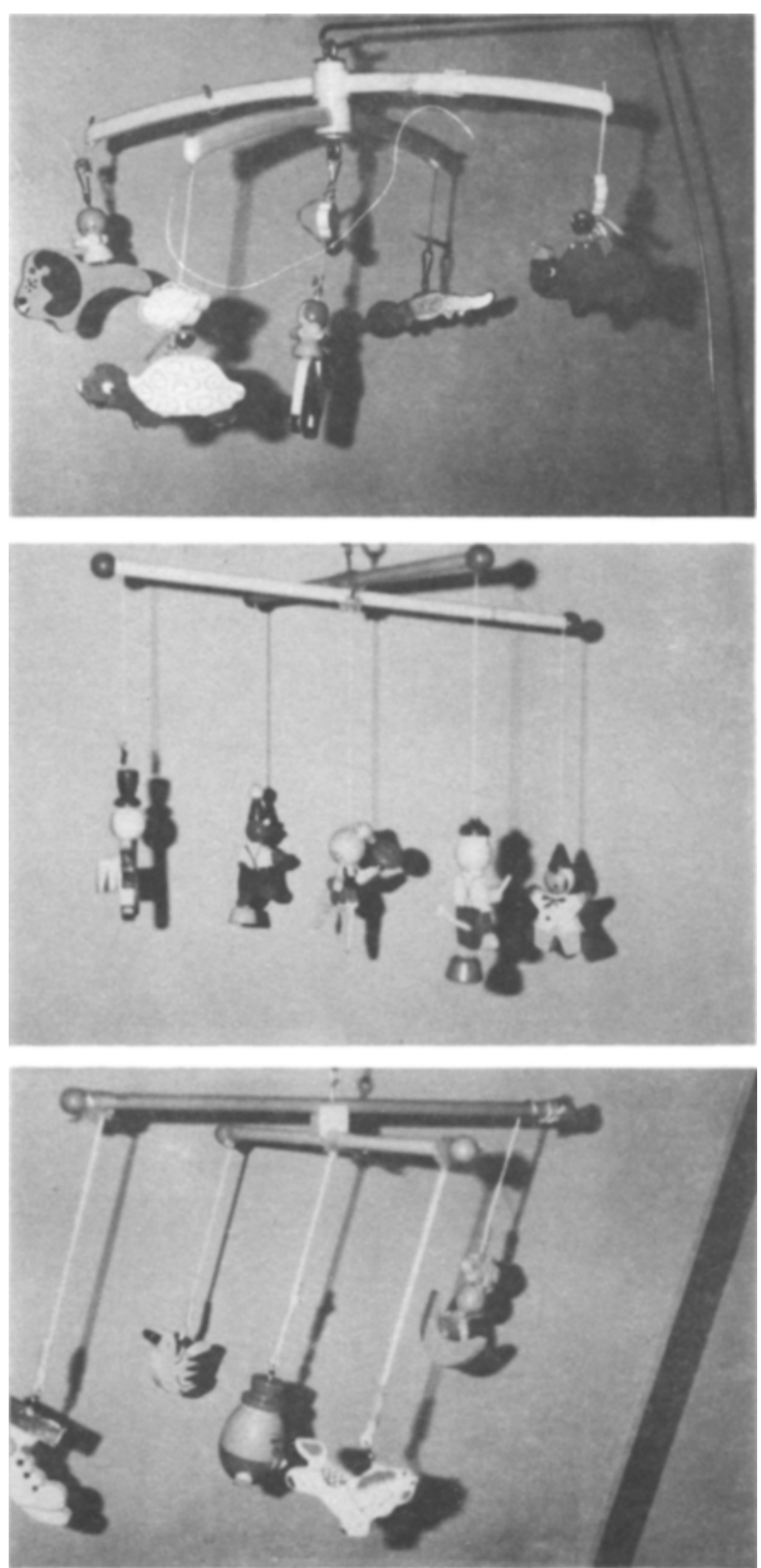

Figure 1. The three mobile models that served as the cues with which 3-month-old infants were trained and/or tested in Experiments 1-5.

head suspension bars protruded toward the center of the crib. A mobile was suspended from the hook of either bar approximately $24.4-30.5 \mathrm{~cm}$ above the infant's chest. A white satin ribbon was looped around the infant's right ankle and was connected without slack to one of the overhead suspension bars; a mobile was hung from the suspension bar nearest the experimenter. During reinforcement phases, the ribbon was connected to the same suspension bar as the mobile, with the result that each kick activated the mobile at a rate and with an intensity proportional to the rate and intensity of kicking. During nonreinforcement phases, the ribbon was connected to the suspension bar most distant from the experimenter; in this arrangement, the mobile remained in view, but infants' kicks could not activate it. 
Procedure. The general procedure used with 3-month-olds was analogous to the yes/no recognition task that has been used in studies of postevent-information effects with adults (e.g., Belli, 1989; Tversky \& Tuchin, 1989). However, because infants lack a verbal response to indicate whether or not they recognize an object, we first had to teach them a motoric one-an operant footkick-and then tested them in a go/no-go paradigm in which they produced the motoric response if they recognized the test object and withheld the response if they did not.

The infants were tested supine in their home cribs at a time of day when their mothers thought they were likely to be playful and alert. This time differed from infant to infant but remained relatively constant across sessions for a given infant. The training and testing paradigm is illustrated in Figure 2 . The infants received two 15-min training sessions (one per day) and a procedurally identical test session $24 \mathrm{~h}$ later. Each session began after the ankle ribbon was attached, when the stationary mobile was suspended over the infant for $3 \mathrm{~min}$. In Session 1, this nonreinforcement period was a baseline phase in which the infant's unlearned activity level, or operant level, was ascertained. In Session 3, the initial nonreinforcement period served as the long-term retention test during which the infant's retention or transfer (depending upon whether the test object was the same or different, respectively) was recorded. Next followed a 9-min reinforcement phase (acquisition) during which the ankle ribbon was moved to the same hook as the mobile, and the infant's kicks were conjugately reinforced by mobile movement. Finally, each session ended as it had begun, with a 3-min nonreinforcement period during which the ankle ribbon was returned to the inactive, or "empty," stand. In Session 2, this served as the immediate retention test during which the infant's final level of acquisition was assessed after zero delay.

Following the 3-min long-term retention/transfer test in Session 3, reinforcement was again introduced to ensure that the infants who failed to respond during the initial long-term test were not unmotivated, ill, or otherwise incapable of responding on that particular day. We emphasize that all measures of retention were obtained during periods when the stationary mobile and the ankle ribbon were attached to different stands. In this way, all measures of retention reflected only what the infant brought into the session from his or her prior experience and not new learning or savings at the time of testing.

The infants were trained for 2 days with either the same mobile (constant training) or a different one (variable training) in each session and were tested 1 day later with either their Session 1 mobile (Groups AA/A and AB/A) or a novel one (Groups AA/B and $A B / C)$. The letters preceding and following the slash in the group labels represent the mobiles used during the two training sessions and the test session, respectively.

A trained observer, positioned out of the infant's direct line of sight, recorded the number of times per minute that the infant kicked the foot with the ribbon attached. A second observer, also stationed out of the infant's direct view, independently recorded kicks/minute for $\mathbf{4 4 3}$ min during randomly selected sessions of 26 infants across all experiments. A Pearson product-moment correlation computed over their joint response counts/minute yielded a coefficient of .98 .

A kick was defined as any horizontal or vertical movement of the leg that at least partially retraced its arc of excursion in a smooth, continuous motion (Rovee \& Rovee, 1969). Only infants whose response rate increased 1.5 times or more above their baseline level in 2 of any 3 consecutive minutes during an acquisition phase (the learning criterion) were retained for retention testing in Session 3.

\section{Results and Discussion}

The kicks/minute of each infant were averaged across 3-min blocks and subjected to one-way analyses of variance (ANOVAs) to determine whether the groups differed either prior to (baseline phase: Block 1, Session 1) or following (immediate retention test: Block 5, Session 2) training. They did not differ, despite the fact that two groups were trained with the same mobile in each session and two were trained with different ones.

Performance during the 24-h retention/transfer test was assessed in terms of two individual measures of relative responding that we have used in all of our previous studies of retention and transfer (for review, see Rovee-Collier \& Hayne, 1987). For each infant, a retention ratio was calculated by dividing the infant's mean kick rate during the 3-min long-term retention test at the outset of Session 3 (test session) by the same infant's mean kick rate during the 3-min immediate retention test at the end of Session 2. The resulting ratio indicates what proportion of the infant's performance after zero delay, when reten-

NONREINFORCEMENT PHASE: Mobile is stationary

REINFORCEMENT PHASE: Mobile moves in response to infant kicking

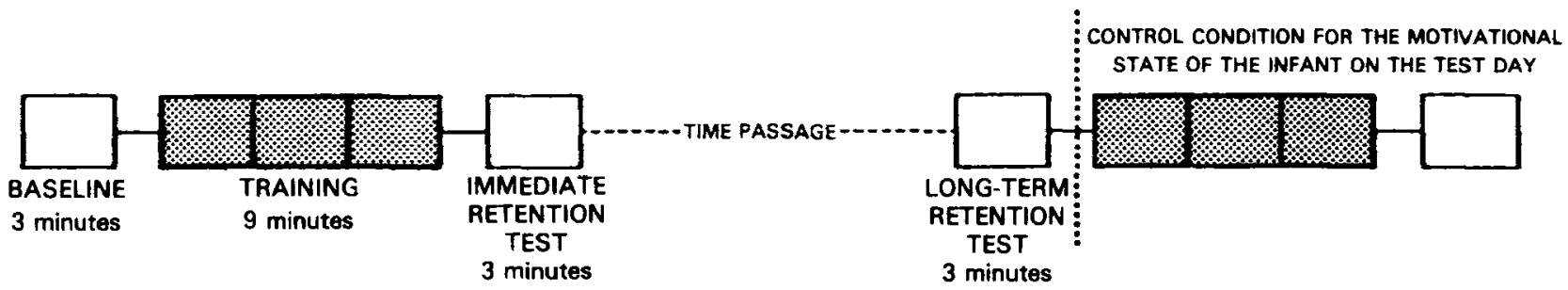

Figure 2. The standard training and retention test paradigm. Infants received two identical training sessions; the long-term test occurred $24 \mathrm{~h}$ after Session 2 in Experiments 1, 2, 3, and $4 B, 48$ h after Session 2 in Experiment 4A, and 14 days after Session 2 in Experiments $5 \mathrm{~A}$ and $5 \mathrm{~B}$. Retention is measured during nonreinforcement periods at the conclusion of training (the immediate retention test) and again after a specified delay (the long-term retention test). Retention is inferred if responding during the long-term test is greater than responding during the initial baseline phase in Session 1; the degree of forgetting is measured in terms of the response decrement from the immediate retention test in Session 2 to the long-term test. 
tion should be best, is still expressed $24 \mathrm{~h}$ later during the long-term test. A retention ratio greater than or equal to 1.00 indicates perfect retention; the lower the retention ratio, the poorer the degree of retention. A group mean retention ratio significantly less than a hypothetical population mean retention ratio of 1.00 (perfect retention) indicates that significant forgetting has occurred.

Because individual infants achieve different final performance levels during training, the absolute value of a mean retention ratio significantly less than 1.00 is not informative as to whether a group's forgetting is partial or complete. To answer this, it is necessary to compute a second relative measure based on each infant's initial baseline response rate, the baseline ratio. This ratio is calculated by dividing each infant's mean kick rate during the 3-min long-term retention test at the outset of Session 3 by that same infant's mean kick rate during the 3-min baseline phase at the outset of Session 1 (operant level). The baseline ratio expresses the extent to which each infant's mean kick rate during the long-term retention test exceeds that same infant's baseline rate. A baseline ratio of 1.00 indicates that test performance is not above operant level (no retention); a group baseline ratio significantly greater than a hypothetical baseline ratio of 1.00 indicates that the group exhibited significant retention.

A one-way ANOVA over the baseline ratios of the four groups indicated that they differed significantly $[F(3,20)$ $=8.26, p<.0009$ ]. A Tukey's Studentized range test indicated that the mean baseline ratios of Groups AA/A and $A B / C$ did not differ and that both were significantly higher than those of groups $A A / B$ and $A B / A$, which also did not differ.

Although the ANOVA revealed that groups differed, it did not answer the question of primary interest-that is, whether or not any groups exhibited significant retention or transfer. Even though the groups in the present study differed, all may have exhibited retention/transfer, or none may have. To answer this, therefore, we used directional $t$ tests to compare the baseline ratio and retention ratio of each group against the corresponding theoretical population ratios of 1.00 (no-retention/transfer and no-forgetting/complete transfer, respectively). The mean baseline ratios of all groups are presented in Figure 3. Infants who were both trained and tested with either the same (Group $A A / A$ ) or a novel (Group $A B / C$ ) mobile each day exhibited excellent retention or transfer, respectively, $24 \mathrm{~h}$ later. Their baseline ratios were significantly greater than a theoretical baseline ratio of 1.00 [Group $\mathrm{AA} / \mathrm{A}, t(5)=3.01, p<.025 ;$ Group $\mathrm{AB} / \mathrm{C}, t(5)=3.71$, $p<.01]$, and their retention ratios $(M=.77, S E=.08$, and $M=.98, S E=.09$, respectively) were not significantly less than a theoretical retention ratio of 1.00 .

In contrast, infants who were trained with the same mobile for 2 days and tested with a novel one (Group AA/B) exhibited no transfer whatsoever. Their baseline ratio was not significantly greater than a theoretical value of 1.00 , and their retention ratio $(M=.53, S E=.17)$ was significantly less than a theoretical value of $1.00[t(5)=2.70$,

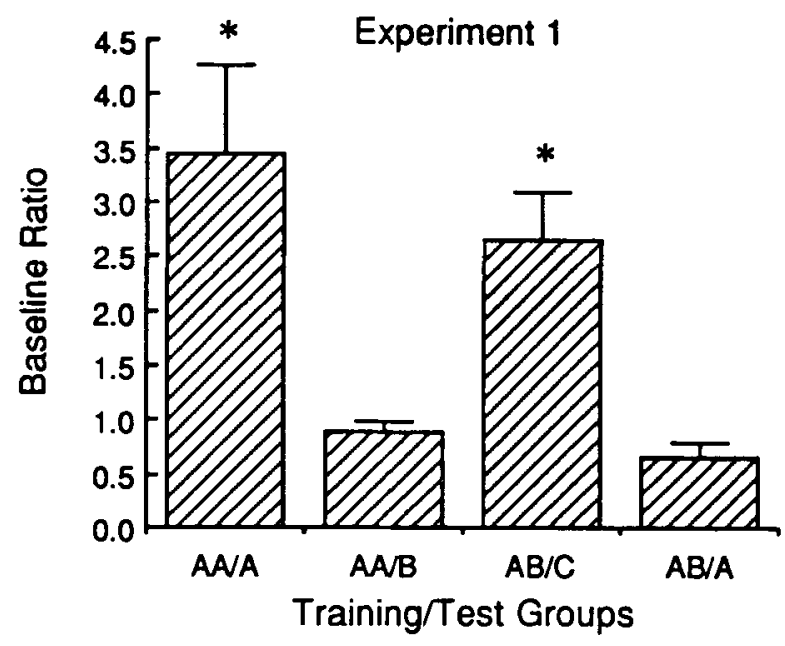

Figure 3. Mean baseline ratios of independent groups $(n=6)$ who were trained either with the same mobile for two sessions (constant training) or with a different one in each session (variable training) and were then tested either with the familiar, Session 1 mobile or with a different mobile $24 \mathrm{~h}$ later. The two letters before the slash indicate the mobile used in each training session; the letter after the slash indicates the test mobile. An asterisk indicates that a group exhibited significant retention (i.e., $M$ baseline ratio significantly above a theoretical population baseline ratio of 1.00$)$. Vertical bars indicate $\pm 1 S E$ (Experiment 1).

$p<.025]$. Importantly, this result confirms that the infants could discriminate between the different mobile models to be used in all succeeding experiments.

Finally, infants who were trained with a novel mobile in each of two sessions but were tested with the familiar Session 1 mobile (Group AB/A) also performed poorly during the 24-h test. Their baseline ratio was not significantly above the theoretical baseline value of 1.00 , and their retention ratio $(M=.61, S E=.18)$ was significantly below the theoretical retention ratio of $1.00[t(5)=$ $2.12, p<.05]$. Although the poor test performance of infants in this group was surprising because they had been explicitly trained with mobile A for an entire session, it was not unexpected; the same result has surfaced in three previous studies involving a variable-training procedure (Amabile \& Rovee-Collier, 1991; Boller \& Rovee-Collier, 1992; Fagen, Morrongiello, Rovee-Collier, \& Gekoski, 1984).

One interpretation of this perplexing but consistent result is that the memory attributes representing the Session 1 mobile (A) were subsequently overwritten or displaced by those representing the Session 2 mobile (B), with the effect that the $A B / A$ group became a $B B / A$ group for which the test mobile was now novel. Had this been the case, however, Group $\mathrm{AB} / \mathrm{C}$, which was identically trained, should have enjoyed a similar fate and been converted into a $\mathrm{BB} / \mathrm{C}$ group. Under these conditions, infants in this group should also have performed poorly during testing with a novel mobile, but they did not. Their test performance was excellent. We conclude, therefore, that the memory representation of the Session 1 mobile was 
not overwritten or displaced in Session 2 but remained intact. Rather, the inability of infants in group $A B / A$ to recognize their Session 1 training mobile must have resulted from interference with the retrieval process at the time of testing in Session 3 (Ceci, Ross, \& Toglia, 1987; Murnane \& Shiffrin, 1991).

\section{EXPERIMENT 2}

In the preceding experiment, only infants who were explicitly trained with a different mobile in each of two sessions (variable training) transferred responding to another different test mobile $24 \mathrm{~h}$ later. Although infants who were trained with the same mobile in each session (constant training) recognized that particular mobile $24 \mathrm{~h}$ later, they failed to respond if their test mobile was different. The data of these groups, therefore, provided the standard against which the effects of postevent information in the succeeding experiments were compared.

In Experiment 2, we asked whether briefly and passively exposing constantly trained infants to information about a novel mobile after training was over would influence their performance during a retention/transfer test $24 \mathrm{~h}$ later. If the infants subsequently produced the learned response to a novel test mobile in the same fashion as infants in Experiment 1 who had been explicitly trained with a discriminably different mobile in each session (Group $A B / C$ ), then this would indicate that the information to which infants were exposed after training was over had been integrated with the previously established training memory.

Although Greco et al. (1990) had found that the attributes of a novel object were not integrated with the prior training memory unless it was exposed in motion-a function it shared with the category-training exemplars-we thought that information about shared function might not be necessary because the novel mobile to which infants would be passively exposed more closely resembled the training mobile than that used in the earlier study.

In the final design, therefore, the infants were trained with the same mobile for two sessions and were then allowed merely to observe a novel, motionless mobile immediately after training was over. Independent groups were tested $24 \mathrm{~h}$ later with their original training mobile, the briefly and passively exposed novel mobile, or a completely novel one.

\section{Method}

Subjects. Eighteen infants ( 8 males, 10 females; $M=91.2$ days, $S D=5.0$ ), recruited as before, were randomly assigned to three independent groups $(n=6)$ as they became available for testing. Additional infants were excluded as a result of crying $(n=7)$ or inattention to the mobile $(n=2)$ for more than 2 consecutive minutes in any of the three sessions, failing to meet the initial learning criterion $(n=4)$, or illness $(n=2)$.

Apparatus and Procedure. The mobiles and apparatus were the same as in Experiment 1; mobile models were again counterbalanced within and across groups during training and testing.

Training and testing procedures were identical to those received by the constant-training groups in Experiment 1 . The infants in the present study, however, were briefly exposed to a novel mobile during a postevent-information procedure at the end of training in Session 2. They were tested $24 \mathrm{~h}$ later with the training mobile (Group AAb/A), the exposure mobile (Group AAb/B), or a completely novel mobile (Group $A A b / C$ ). The lowercase letter in the group label designates the mobile that was exposed during the postevent-information procedure.

The postevent-information procedure lasted only $3 \mathrm{~min}$ and followed the immediate retention test in Session 2. At this time, the training mobile and the ribbon connecting the infant's ankle to the mobile stand were removed, and a novel mobile was suspended motionless from the stand where the training mobile had hung. After $3 \mathrm{~min}$, the experimenter removed the mobile from the stand, and the procedure was over.

\section{Results and Discussion}

Separate one-way ANOVAs over the mean response rates during the baseline phase (Session 1, Block 1) and the immediate retention test (Session 2, Block 5) indicated that the groups did not differ in either unlearned activity or final level of training, respectively, prior to the introduction of the postevent-information procedure.

The mean baseline ratios of the three groups are plotted in Figure 4. An ANOVA over these ratios indicated that they did not differ. Directional $t$ tests comparing each group's baseline and retention ratios against the corresponding theoretical values of 1.00 revealed that only infants in Group AAb/A exhibited significant retention. Their baseline ratio was significantly greater than 1.00 $[t(5)=3.77, p<.01]$, but their retention ratio $(M=$ $.79, S E=.08)$ was significantly less than $1.00[t(5)=$ $2.70, p<.025$ ], indicating that performance was lower during the long-term test than during the immediate retention test $24 \mathrm{~h}$ earlier. In contrast, neither Group AAb/B

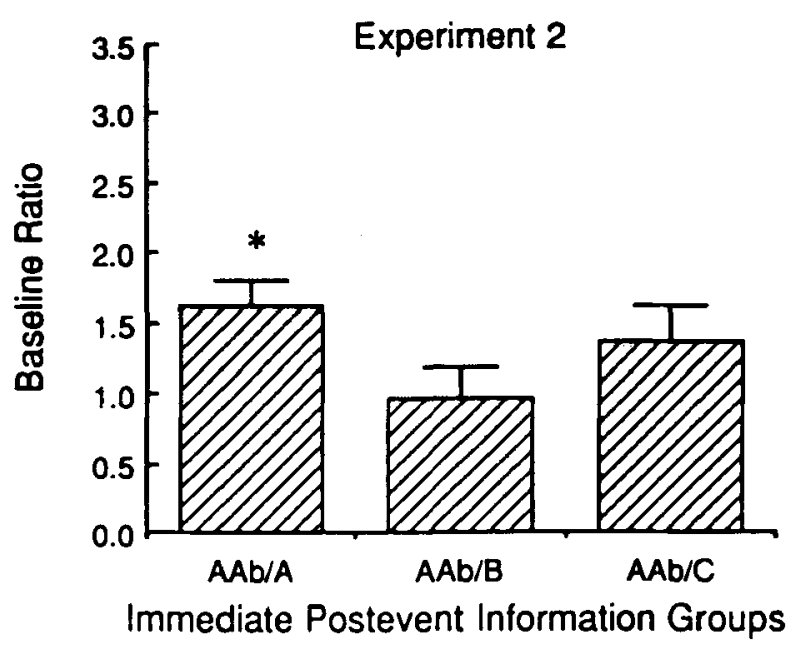

Figure 4. Mean baseline ratios of infants trained with the same mobile for two consecutive sessions, exposed to a motioniess novel mobile for 3 min at the end of the second session, and tested with either the exposed mobile (Group AAb/B) or a completely novel one (Group AAb/C) 24 h later. Neither group exhibited retention (i.e., $M$ baseline ratios not significantly $>1.00$ ). The lowercase letter represents the mobile that was exposed during the postevent-information procedure. Vertical bars indicate $\pm 1 S E$ (Experiment 2). 
nor Group $\mathrm{AAb} / \mathrm{C}$ responded above baseline during testing (i.e., $M$ baseline ratios not significantly $>1.00$ ), and both mean retention ratios were either significantly less than the corresponding theoretical value of 1.00 [Group $\mathrm{AAb} / \mathrm{B}, M=.41, S E=.11, t(5)=5.32, p<.005]$ or approached being so [Group AAb/C, $M=.76, S E=$ $.14 ; t(5)=1.69, p<.08]$, with a mean value slightly lower than the mean retention ratio of Group AAb/A. We conclude, therefore, that the infants' brief and passive postevent exposure to the stationary novel mobile did not affect their prior training memory.

\section{EXPERIMENT 3}

In the preceding experiment, contrary to our original expectations, postevent information about the physical characteristics of a novel but stationary mobile did not affect the infants' prior memory of training even though it was temporally contiguous with training. The same finding had been obtained by Greco et al. (1990) when, following variable training, they had exposed infants to a stationary object that bore no physical resemblance to the training mobiles. Greco et al. found that the highly physically dissimilar object was integrated with the prior training memory, however, if they exposed infants to information about the object's function (movement), which it shared with the prior training exemplars.

In Experiment 3, therefore, we expanded the postevent information to include information that the novel mobile could function like the original training mobile-that is, it could move.

\section{Method}

Subjects. Eighteen infants (8 males, 10 females) with a mean age of 88.6 days ( $S D=5.6$ ) were recruited as before. As they became available for testing, the subjects were randomly assigned to one of three postevent-information groups differentiated in terms of whether testing was with the training mobile (Group $A A b / A$ ), the novel exposure mobile (Group AAb/B), or a completely novel mobile (Group $\mathrm{AAb} / \mathrm{C})$. Additional subjects were excluded for crying in excess of 2 consecutive minutes in any session $(n=5)$, illness $(n=2)$, or a scheduling conflict $(n=3)$.

Apparatus and Procedure. All aspects of apparatus and procedure were identical to those of Experiment 2 except that the ribbon was reconnected to the mobile stand after the novel exposure mobile was in place, and the experimenter noncontingently drew and released its other end for $3 \mathrm{~min}$ at a frequency that matched the frequency with which the infant had kicked to move the training mobile in each of the final $3 \mathrm{~min}$ of acquisition in Session 2. In this way, the functional postevent information about the novel mobile was phenomenologically equivalent to the movement that each infant had previously witnessed in the original mobile during training.

\section{Results and Discussion}

As before, one-way ANOVAs over the mean response rates during the baseline phase and the immediate retention test indicated that the three independent groups did not differ either prior to or following training, respectively.
The baseline ratios of all groups are presented in Figure 5 (top panel). Another ANOVA indicated that the mean baseline ratios of the three test groups differed significantly $[F(2,15)=6.96, p<.007]$. A Tukey's Studentized range test indicated that the baseline ratio of Group $\mathrm{AAb} / \mathrm{A}$ was significantly lower than those of Groups $A A b / B$ and $A A b / C$, which did not differ.

Directional $t$ tests between each group's mean baseline and retention ratios and the corresponding theoretical

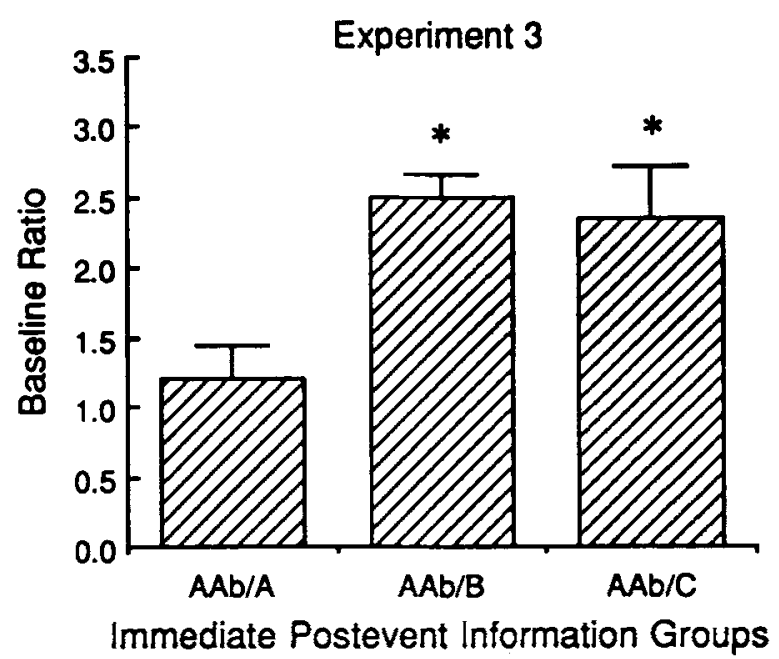

\section{Experiment 4A}

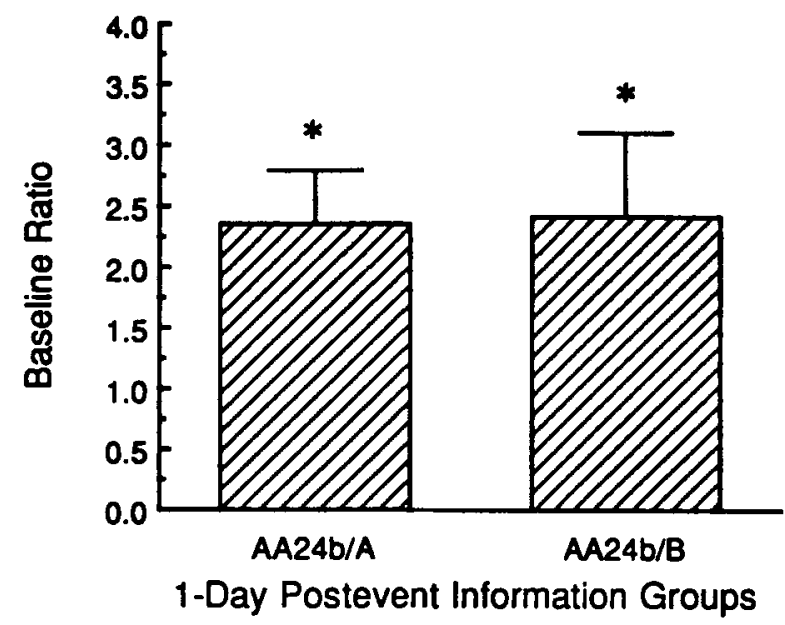

Figure 5. Top panel: Mean baseline ratios of infants trained with the same mobile for two sessions, exposed to a novel mobile moving noncontingently for $3 \mathrm{~min}$ as soon as the second training session was over, and tested with the original mobile (Group AAb/A), the exposed mobile (Group AAb/B), or a completely novel mobile (Group $\mathrm{AAb} / \mathrm{C}$ ) $24 \mathrm{~h}$ later (Experiment 3). Bottom panel: Mean baseline ratios of Group $A A 24 b / A$ and Group $A A 24 b / B$ whose experimental procedures were identical to those of the corresponding groups in Experiment 3 except that $24 \mathrm{~h}$ intervened between the end of the second training session and the postevent exposure to the novel mobile (Experiment 4A). An asterisk indicates that a group exhibited significant retention (i.e., $M$ baseline ratio significantly $>1.00$ ). Vertical bars indicate $\pm 1 S E$. 
population ratios of 1.00 indicated that infants who were passively exposed to the novel mobile in motion after the end of training not only responded to that mobile during the 24-h test but also to a novel mobile that they had not previously seen. The baseline ratios of Groups AAb/B and $\mathrm{AAb} / \mathrm{C}$ significantly exceeded $1.00[t(5)=2.50, p<$ .0005 , and $t(5)=3.68, p<.01$, respectivelyl, and the retention ratios of both groups $(M=.92, S E=.12$, and $M=.90, S E=.08$, respectively) were not significantly less than 1.00. The latter result is consistent with the data of Group $\mathrm{AB} / \mathrm{C}$ in Experiment 1, as well as with those of Greco et al. (1990), who had explicitly trained infants with a novel mobile in each session, and suggests that the memory representation of the training mobile of Session 2 (but not of Session 1) was recoded in terms of the mobile to which infants were exposed during the posteventinformation procedure immediately following that second session.

In contrast, infants who were tested with their original training mobile (Group AAb/A) exhibited no retention. Their baseline ratio was not significantly above 1.00 , and their retention ratio $(M=.66, S E=.12)$ was significantly less than $1.00[t(5)=2.78, p<.025]$. Because Groups $A A b / B$ and $A A b / C$ exhibited excellent retention, however, we attribute the poor retention of Group $A A b / A$ to retroactive interference at the time of retrieval (see also Murnane \& Shiffrin, 1991). The finding that conflicting postevent information impairs recognition of a prior event is a typical finding in conventional studies of eyewitness testimony with adults (Lindsay, 1990; E. F. Loftus, 1979, 1981) and children (Ceci, Toglia, \& Ross, 1987; but see Zaragoza, 1991). The similarity between the test performance of Group $A A b / A$ in Experiment 3 and Group $A B / A$ in Experiment 1 provides additional support for our conclusion that the memory representation of the training mobile in Session 2 was recoded in terms of the novel mobile to which infants were exposed at the end of Session 2 , when training was over.

The data of all three groups, considered jointly, provide strong evidence that postevent information is integrated with infants' memory representation of prior training and influences their subsequent retention. Notably, although integration of the novel object with the prior training memory did not occur unless the postevent exposure included information about a function (i.e., movement) that the novel mobile shared with the training mobile, this common functional information was not present at the time of retention testing, when the novel mobile was stationary. From this, we conclude that the common, functional information acted as a catalyst that integrated the representation of the novel object with that of the training event in working or active memory. Once the integration had occurred, however, the catalyst was unnecessary for subsequent memory operations.

Although we used the term "integration" to describe how postevent information modified infants' previously established memory representation, the nature of the in- tegration was unclear. Did information about the novel mobile replace previously stored information about the training mobile in the original memory? Did the integration result in a new memory that contained a blend of old and new information? These questions were addressed in Experiments $4 \mathrm{~A}$ and $4 \mathrm{~B}$.

\section{EXPERIMENT 4A}

In Experiment 1, the memory attributes representing the Session 1 mobile had not been overwritten by the novel training mobile in Session 2. In Experiment 3, however, the representation of the Session 2 training mobile did appear to be overwritten by the novel mobile to which the infants were exposed at the end of Session 2. These findings suggest that a memory representation can be overwritten by conflicting information that is encountered while the memory is in an active state (i.e., in primary or working memory). The fact that the Session 1 memory of mobile A in Experiment 1 had not been overwritten by mobile $B$ when it was retrieved into primary memory in Session 2, however, suggests that only a copy of the Session 1 memory was retrieved, while the original remained intact.

We think these differences point to the necessity of distinguishing between memory tokens and memory types. This distinction is modeled after Treisman's analysis of the formation and retrieval of object tokens, which are memory representations of perceived objects (Kahneman \& Treisman, 1984; Kahneman, Treisman, \& Gibbs, 1992; Treisman, 1992). Although Treisman distinguished between object files, object tokens, and object types, our adaptation includes an entire event rather than a single object that subjects perceive during an event or episode. By this account, each time a copy of one or more memory tokens of an event is retrieved into primary memory, a new token of that type of event (an episodic memory) is created. Retrieved information will be updated or displaced by conflicting postevent information that is physically present and active in primary memory with the retrieved information. As a result, the newly created memory token will reflect the final status of the current event. After this new memory token enters long-term memory, it is thereafter buffered against modification-only a copy of it can subsequently be retrieved and modified.

This account predicts that delaying infants' exposure to novel postevent information until the original token has entered long-term memory will protect it from modification. Instead, the postevent information should retrieve and modify a copy of the original token, creating a new, updated memory token. We tested this prediction in Experiment 4A.

\section{Method}

Subjects. The subjects were 12 infants ( 4 males, 8 females), with a mean age of 89.7 days $(S D=3.8$ ). They were randomly distributed into two groups (Groups AA24b/A and AA24b/B) as they be- 
came ready for study. Additional subjects were excluded from the final sample for crying longer than 2 consecutive minutes in any session $(n=1)$, failing to meet the learning criterion $(n=1)$, or illness $(n=1)$.

Procedure. All aspects of procedure were the same as in Experiment 3 except that $24 \mathrm{~h}$ elapsed between the end of Session 2 and the postevent-information procedure. As before, the retention interval between the postevent-information procedure and testing was 1 day; this meant that 2 days elapsed between training and testing.

\section{Results and Discussion}

One-way ANOVAs over the response rates during the baseline phase and the immediate retention test indicated that Groups AA24b/A and AA24b/B did not differ either prior to or after training, respectively. A one-way ANOVA over their mean baseline ratios also yielded no difference.

Directional $t$ tests between the mean baseline and retention ratios of each group and the corresponding theoretical population ratios of 1.00 indicated that both groups performed well during the long-term test. The baseline ratios of the two groups are shown in Figure 5 (bottom panel). Both baseline ratios were significantly greater than 1.00 [Group AA24b/A, $M=2.41, t(5)=2.52, p<.05$; Group AA24b/B, $M=1.86, t(5)=3.82, p<.01]$, and neither retention ratio was significantly less than 1.00 $(M=.81, S E=.13$, and $M=.76, S E=.15$, respectively).

The excellent retention exhibited by Group AA24b/A confirms that information in a memory token is not displaced by postevent information unless it is in an active state at the time conflicting information is presented. Rather, once a memory token has entered long-term memory, it appears to be protected against overwriting. On the other hand, the significant retention exhibited by Group AA24b/B indicates that postevent information can still be integrated with the prior training memory in a new memory token despite a 1-day delay between training and the postevent-information procedure. These data suggest that the original mobile and the passively exposed one are represented in different memory tokens. Presumably, the presence of $t w o$ memory tokens containing mobile A in long-term memory increased the probability that a token containing mobile A would be retrieved at the time of testing. Also according to this account, frequency-ofencounter information, which is thought to be automatically processed (Hasher \& Zacks, 1979), would reflect the number of different tokens of each memory type.

Although the present data appear to contradict evidence that the impact of postevent information in adults is greatest when that information is introduced after a delay rather than immediately (Belli, Windschitl, McCarthy, \& Winfrey, 1992; E. F. Loftus et al., 1978; see also E. F. Loftus, 1979), this is not the case. Rather, the present data indicate that overwriting can occur whenever new and old information cohabit active memory, before formation of the new memory token is complete. In fact, in a sequel to the Greco et al. (1990) study, we have found that new postevent information can be integrated with in- fants' prior memory tokens for as long as 4 days after category training. Consistent with the findings of Belli et al. (1992) and Loftus et al. (1978), the impact on retention was greater when the postevent information was introduced at the end of this time window rather than immediately after the final session of category training (Rovee-Collier, Greco-Vigorito, \& Hayne, in press).

\section{EXPERIMENT 4B}

In the preceding experiment, when a 24-h delay intervened between the end of training and the introduction of the postevent information, infants' recognition of the original mobile was not impaired as it had been for Group $\mathrm{AAb} / \mathrm{A}$, whose postevent information had been presented immediately after the end of training in Experiment 3. Our preferred interpretation for the lack of impairment was that the postevent information had retrieved the memory tokens of the past training sessions into working memory, with the result that a new memory token was formed in which the passively exposed novel mobile replaced the original one.

Alternatively, however, in Experiment 3, postevent information about the novel mobile may have blended with the information about the original mobile without displacing it. By this account, the new memory that was created would have contained elements of both mobiles (e.g., E. F. Loftus \& Hoffman, 1989). If this was the nature of the integration that occurs, then infants in Group $\mathrm{AAb} / \mathrm{A}$ should exhibit retention if they are tested with a mobile composed of both old and new elements. This alternative was tested in Experiment 4B.

\section{Method}

Subjects. Six infants ( 2 males, 4 females) were recruited as before and assigned to Group $\mathrm{AAB} / \mathrm{A}+\mathrm{B}$. The infants had a mean age of 90.5 days $(S D=5.7)$ on the first day of training.

Apparatus and Procedure. All aspects of training, the posteventinformation procedure, and testing were the same as for Group $\mathrm{AAb} / \mathrm{A}$ in Experiment 3 except that mobiles $\mathrm{A}$ and $\mathrm{B}$ contained six objects each, and the test mobile contained three objects from mobile $\mathrm{A}$ and three objects from mobile $\mathrm{B}$.

\section{Results}

Despite the fact that their test mobile contained equal numbers of objects from the training mobile and the novel exposure mobile, the infants failed to recognize it during the 24-h test. Their baseline ratio $(M=1.47, S E=.24)$ was not significantly greater than a theoretical baseline ratio of 1.00 , indicating that responding did not exceed operant level; their retention ratio was significantly less than a theoretical retention ratio of $1.00[M=.77, S E=$ $.11, t(5)=2.05, p<.05]$, indicating a significant retention deficit. In all respects, their performance mirrored that of Group AAb/A in Experiment 3 (see Figure 5, top panel). In previous research with the same mobiles but in which no postevent information was introduced, infants had similarly failed to recognize their training mobile if more than a single novel object had been substituted into 
it (Hayne, Greco, Earley, Griesler, \& Rovee-Collier, 1986; Rovee-Collier, Patterson, \& Hayne, 1985). The considerable specificity required of effective retrieval cues at this age, therefore, encourages us to reject the memoryblend alternative: Had the test mobile matched the representation in long-term memory, infants would have recognized it.

\section{EXPERIMENT 5A}

In the preceding experiments, we showed that novel postevent information could influence infants' retention of a prior event for 1 to 2 days. Whether the postevent information remains part of the memory representation after a substantially longer delay is unknown, although recent evidence suggests that it might (Boller \& RoveeCollier, 1991). We have previously shown that after delays so long that the memory has been forgotten, it can still be recovered if, several hours prior to the long-term retention test, infants are briefly exposed to a cue that had been encoded in the original memory representation $(\mathrm{Fa}$ gen \& Rovee-Collier, 1983; Rovee-Collier, Sullivan, Enright, Lucas, \& Fagen, 1980; Sullivan, 1982; for review, see Rovee-Collier \& Hayne, 1987). This cue or reminder primes the latent or dormant memory, increasing its accessibility in an all-or-none fashion. Whether or not the memory was reactivated is confirmed during a standard delayed-recognition test $24 \mathrm{~h}$ later.

Because only cues that were originally encoded in the memory can serve as reminders for it, the original training mobile is a highly effective reminder, but a novel mobile-or even a mobile that has been only slightly altered from its original form-is not (Rovee-Collier et al., 1985 ) unless infants had been variably trained at the outset (Hayne et al., 1987; Greco et al., 1990; Shields \& Rovee-Collier, 1992).

We reasoned that if postevent information about the novel mobile is a relatively stable component of the memory representation with which it has been integrated, then the novel mobile should be an effective reminder for the training memory. On the other hand, if it is only a transient component of the memory representation, then it should not. Experiment 5A examined these possibilities.

\section{Method}

Subjects. Eighteen infants ( 10 males, 8 females; $M$ age $=89.1$ days, $S D=5.6$ ) were assigned to one of three groups $(n=6)$ as they became available for testing. Additional infants were excluded for crying for longer than 2 consecutive minutes in any session $(n=$ $5)$ or a scheduling conflict $(n=1)$.

Procedure. All aspects of training, testing, and the posteventinformation procedure were identical to those of Experiment 3 . In addition, all infants received a reactivation treatment that consisted of a brief exposure to either the original training mobile (Group $\mathrm{AAb}-\mathrm{A} / \mathrm{A}$ ) or the novel mobile (Group AAb-B/A) being moved noncontingently 13 days after the second training session-a delay after which forgetting is complete for infants of this age. During the reactivation treatment, the infant was placed in a sling seat inside its crib with the reminder mobile suspended overhead. One end of a ribbon was attached to the same hook as the mobile. The other end was drawn and released for 3 min by the experimenter, squatting out of sight, at the same rate that the infant had kicked in each of the last $3 \mathrm{~min}$ of acquisition in Session 2 . When $3 \mathrm{~min}$ had elapsed, the experimenter removed the mobile from the stand, the mother lifted the infant out of the seat, and the reactivation treatment was over.

Twenty-four hours after the reactivation treatment (i.e., 14 days after Session 2), both groups were tested with the original training mobile. To control for the physical discrepancy between the reminder and the test mobile, a third group was both reminded and tested with the novel mobile (Group AAb-B/B). In the group labels, the letter preceding the slash designates the reminder mobile; the letter after the slash again designates the test mobile.

\section{Results and Discussion}

One-way ANOVAs over the mean response rates during the baseline phase and the immediate retention test again indicated that the groups did not differ either prior to or following training. A one-way ANOVA over the mean baseline ratios also yielded no group differences.

Directional $t$ tests revealed that neither the original mobile nor the novel mobile was an effective reminder, regardless of the test mobile. No baseline ratios were significantly above the theoretical baseline ratio of 1.00 , and all retention ratios were significantly less than a theoretical retention ratio of 1.00 [Group AAb-A/A, $M=.49$, $S E=.10, t(5)=5.03, p<.005 ;$ Group AAb-B/A, $M=$ $.41, S E=.09, t(5)=6.55, p<.005 ;$ Group AAb-B/B, $M=.35, S E=.06, t(5)=11.36, p<.0005]$. The mean baseline ratios of all groups are presented in Figure 6 (left panel). Data of infants in a previous study (Rovee-Collier \& DuFault, 1991), who were identically trained, reminded, and tested but who received no postevent information, are presented for comparison.

Although infants who received no postevent information about a novel mobile exhibited perfect retention 1 day after a reactivation treatment with the original training mobile (Group AA-A/A), infants who were briefly exposed to a novel mobile immediately after training exhibited none when reminded with the original training mobile (Group AAb-A/A). Recall that Group AA/A (Experiment 1) had similarly exhibited perfect retention of the newly acquired memory only 1 day after training, when that memory was still highly accessible, but Group AAb/A (Experiment 3) had exhibited none after the same delay. We conclude, therefore, that postevent information about mobile B, which had interfered with the effectiveness of the original training mobile as a retrieval cue during a 24-h retention test for Group AAb/A, continued to be represented in the original training memory after 2 weeks and continued to interfere with the effectiveness of the training mobile (mobile $A$ ) as a retrieval cue. As a result, the original mobile was unable to reactivate the training memory.

A similar reactivation failure had occurred in an earlier study when we had exposed 6-month-olds to a novel context immediately following their second training session and then had presented an otherwise effective reminder (the original mobile) in the novel exposure context 3 weeks later (Boller \& Rovee-Collier, 1992). Infants of this age 

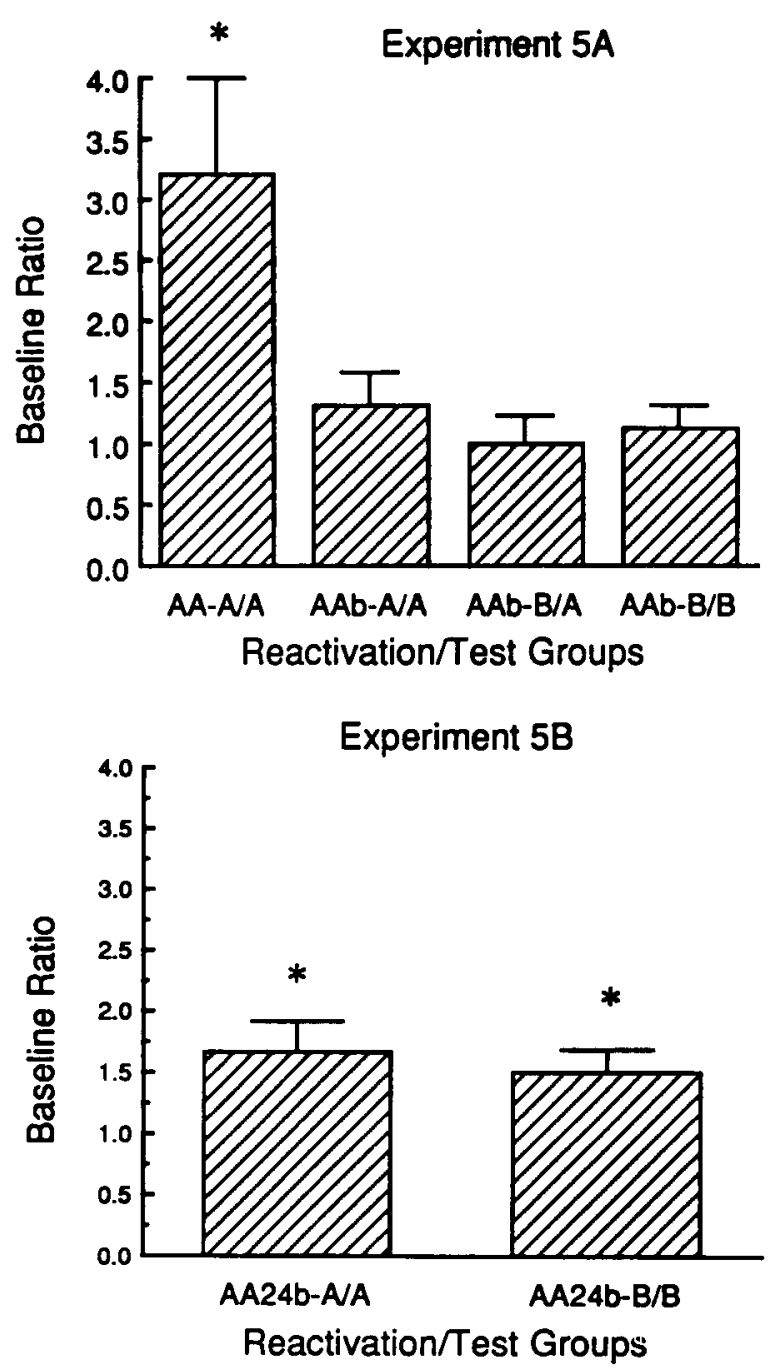

Figure 6. Top panel: Mean baseline ratios of groups whose training, postevent-exposure procedure, and testing were identical to those of Groups $\mathrm{AAb} / \mathrm{A}$ and $\mathrm{AAb} / \mathrm{B}$ in Experiment 3 except that 2 weeks (instead of $24 \mathrm{~h}$ ) intervened between the end of training and the longterm retention test. One day prior to the long-term test, infants received a reactivation treatment either with their original mobile (Group AAb-A/A) or with the novel mobile that was exposed during the postevent-information procedure at the end of Session 2 (Group AAbB/A). A control group was both reminded and tested with the reminder mobile (Group AAb-B/B). None of the reactivation groups exhibited retention (i.e., $M$ baseline ratios not significantly > 1.00). Data from identically trained, reminded, and tested infants (Group AA-A/A) who received no postevent information are shown for comparison (redrawn from Rovee-Collier \& DuFault, 1991). The letter preceding the slash designates the reminder mobile; the letter following the slash designates the test mobile (Experiment 5A). Bottom panel: Mean baseline ratios of two reactivation groups who were treated identically to Group $A A b-A / A$ and Group AAb-B/B in Experiment 5A except that $24 \mathrm{~h}$ intervened between the end of training and the postevent-information procedure (Experiment 5B). In both panels, an asterisk indicates that a group exhibited significant retention (i.e., $M$ baseline ratio significantly $>$ 1.00). Vertical bars indicate $\pm 1 S E$. typically exhibit perfect retention after being reminded in the original training context but none if the reminding context is different. When we exposed infants to the novel context immediately following both Session 1 and Session 2, however, their forgotten memory was successfully reactivated in the novel exposure context (Boller \& RoveeCollier, 1991). Apparently, two memory tokens containing a representation of the novel exposure context were sufficient to override any debilitating effect that might have resulted from competition between equal numbers of memory tokens at the time of retrieval. The preceding result suggests that the reactivation failure in the present experiment may similarly have resulted from a temporary competition between equal numbers of memory tokens at the time of reactivation. This suggestion was explored in Experiment 5B.

\section{EXPERIMENT 5B}

If interference results from a temporary competition between equal numbers of memory tokens at the time of retrieval, then altering the proportion of old:new tokens should eliminate or reduce this competition. As a result, either mobile-new or old-could become an effective reminder. In Experiment 4A, postevent information about the novel mobile was integrated with the original training memory after a 1-day delay without impairing retention of the original mobile. This result suggested that the memory token established in Training Session 2 had remained intact and that the postevent information had been incorporated into a new memory token that was formed when the novel mobile was exposed 1 day later. In Experiment 5B, therefore, we introduced the postevent information after a 1-day delay and asked whether, under these circumstances, either the original mobile or the novel exposure mobile (or both) would reactivate the forgotten training memory.

\section{Method}

Subjects. Twelve infants ( 9 males, 3 females) with a mean age of 87.4 days ( $S E=5.0$ ) on the first day of training were recruited as before. As they became available for study, the infants were randomly assigned to one of two groups that were distinguished by the reminding and test mobile. Group AA24bA/A was reminded and tested with the original training mobile; Group AA24bB/B was reminded and tested with the novel exposure mobile.

Procedure. The infants were treated in all respects like the subjects in Group AAbA/A in Experiment 5A except that their 3-min passive exposure to the novel mobile occurred 1 day after the end of training instead of immediately afterwards.

\section{Results}

When the postevent-information procedure was delayed by 1 day, both the original mobile and the novel exposure mobile were effective reminders for the forgotten memory. The baseline ratios of the two delayed-exposure reactivation groups are shown in Figure 6 (bottom panel). 
Both baseline ratios were significantly greater than a theoretical baseline ratio of 1.00 [Group AA24bA/A, $t(5)=$ $2.57, p<.025$; Group AA24bB/B, $t(5)=2.94, p<$ .025 ], but both retention ratios were also significantly less than a theoretical retention ratio of 1.00 [Group $\mathrm{AA} 24 \mathrm{bA} / \mathrm{A}, M=.61, S E=.07, t(5)=5.82, p<.002$; Group AA24bB/B, $M=.66, S E=.08, t(5)=4.37, p<$ $.005]$. Thus, although both cues now successfully primed the training memory, some competition between the old and new memory tokens was still evident during the delayed recognition test, $24 \mathrm{~h}$ after the memories had been reactivated.

These results offer convergent evidence that postevent information that is presented after a 1-day delay is integrated with the prior memory of training without impairing retention of the original information in that training memory. Presumably, this is accomplished via the creation of another episodic memory token at the time the delayed postevent information is presented rather than by modification of the original memory tokens of prior training sessions. Finally, the fact that the novel exposure mobile could cue retrieval of the training memory 2 weeks later in a reactivation paradigm indicates that the postevent information was an enduring component of that new memory token.

\section{GENERAL DISCUSSION}

The present experiments unequivocally demonstrate that information that infants encounter after an event is over can influence not only their subsequent retention of the details of that original event but also the probability that they will remember the postevent information as having been part of the original event. In both regards, the present findings mirror findings from studies of eyewitness testimony with adults and children who were exposed to misleading postevent information (e.g., Ceci, Toglia, \& Ross, 1987; Chandler, 1991; Lindsay, 1990; E. F. Loftus, 1975, 1979, 1981; E. F. Loftus et al., 1978; Weinberg, Wadsworth, \& Baron, 1983).

Although the striking similarity between these findings may seem surprising in view of the radical differences between the procedures used with infants and those used with linguistically competent subjects, the fundamental paradigms are the same: (1) subjects witness an event, (2) they are introduced to postevent information that conflicts with some aspect of what they previously witnessed, and (3) they are tested for retention of the originally witnessed event and/or for acceptance of the postevent information. The present results suggest that the mechanisms that underlie memory processing may not be fundamentally different in adults and infants. Unlike adults, however, prelinguistic infants are not subject to problems associated with social or task demands, instructional set, and so forth, that often plague conventional research on eyewitness testimony. In addition, their memory performance is not affected by a large network of prior associations. For these reasons, we think that infants are actu- ally the subjects of choice for research on memory modification.

Although much of the early work on adult eyewitness testimony that demonstrated impairment of the original memory by conflicting postevent information has been criticized on methodological grounds (e.g., McCloskey \& Zaragoza, 1985), these criticisms are not relevant to the present experiments. McCloskey and Zaragoza, for example, argued compellingly that recognition tests that force subjects to choose between the original item and the item that had been introduced during the more recent postevent-information procedure biased subjects' responses in favor of the more recent item. They proposed that subjects should be asked instead to choose between the original item and a completely novel one. In the present experiments, however, infants were tested in a go/no-go procedure in which independent groups received only one choice (i.e., one test mobile) that reflected each of these alternatives-the original one, the one that was introduced during the postevent-information procedureas well as a completely novel one.

In fact, after being trained with mobile $A$ and then briefly exposed to mobile $B$, the infants responded to a completely novel mobile, mobile $\mathrm{C}$, which they otherwise would have discriminated. In this instance, postevent information actually facilitated the recognition of a completely novel stimulus whose general features were represented in multiple memory tokens. Presumably, the acquired reliance on general features eliminated the temporary interference seen after $24 \mathrm{~h}$ in Experiment 1 (Group AB/A) and Experiment 3 (Group AAb/A), when the infants were asked to recognize a test stimulus whose specific details were represented in one memory token but not in another. In effect, infants tested with a completely novel mobile behaved as if they had been trained in a categorization procedure-that is, with a different category exemplar in each session (Fagen et al., 1984; Greco et al., 1990; Hayne et al., 1987; Shields \& Rovee-Collier, 1992). Category training, of course, facilitates responding to novel category members but not to novel members of another category.

In studies of adults' eyewitness testimony, subjects reject a completely novel alternative when forced to choose between it and the original item (Belli, 1989). However, adults have never received forced-choice tests between two completely novel objects-one which is a member of the same category as the object that they originally witnessed, and one that is not. If subjects had originally witnessed an accident involving a Volkswagen, for example, and later had received misleading information that the accident had involved a Honda, then they might indicate that the accident had involved a Toyota (a completely novel item) if forced to choose between a Toyota and a truck or bus at the time of testing. Thus, whether postevent information interferes with or facilitates subsequent memory performance depends upon the number and content of prior memory tokens, the alternatives available at the time of retrieval, and the experimenter's definition 
of an appropriate test response in an experimental or reallife situation.

Although the present results do not support McCloskey and Zaragoza's (1985) conclusion that postevent information has no effect on the prior memory representation, they also do not support arguments that postevent information either overwrites the original memory representation (e.g., E. F. Loftus \& G. R. Loftus, 1980) or blends with it in a new memory (E. F. Loftus \& Hoffman, 1989). Instead, the data suggest that postevent information overwrites conflicting information that is coactive with it in primary memory. That is, postevent information that either retrieves a copy of one or more prior memory tokens into primary memory or is encountered while the memory token of a given training event is still being formed (and, hence, still occupies primary memory) can revise or update information with which it conflicts. The final version of this updating process enters long-term memory as a new episodic memory token and thereafter remains intact.

As indicated earlier, this analysis is similar to Triesman's description of the formation of object tokens from object files (Kahneman \& Triesman, 1984; Kahneman et al., 1992; Triesman, 1992). In this account, when an object is initially perceived, an object file is created that reflects the contents of short-term memory. This file includes all new information about the object, including time and place information and the subject's response to it. The object file persists only for as long as the object itself is physically present. Thereafter, it dissipates and is replaced by a permanent object token that consists of the final updáted version of the original object file. When the object is subsequently re-perceived, it retrieves all object tokens with which it shares components into active memoryan individual exemplar account. Object tokens are formed during brief, one-time exposures to a visual stimulus and can still be primed after delays so long that they are no longer recognized in explicit memory tests (Cave \& Squire, 1992; Haist, Musen, Squire, 1991; Musen \& Squire, 1992; Musen \& Triesman, 1990; Triesman, 1992). These reports are consistent with our present finding that a new memory token is formed during a brief one-time exposure to novel postevent information and that it can still be primed after delays when recognition is no longer exhibited (see also Greco et al., 1990; RoveeCollier et al., in press).

In addition to providing insights into the analysis of the varied effects of postevent information on retention, this analysis has general implications for how categories expand and for the special role of functional information in this process (Greco et al., 1990). From a more general perspective, we think that this same process underlies the development of the knowledge base (Rovee-Collier et al., in press). Prelinguistic infants, like children and adults, do not live in a vacuum. Their lives consist of a series of events, most of which they only passively observe. The present data reveal that when a passively observed event contains information that is functionally sim- ilar to information that is already in long-term memory, it may retrieve the relevant memory token(s) and create a new memory token that includes the new information. Not only can the integration of postevent information occur after a substantial delay, but also it appears to be relatively permanent. Although infants consistently treat postevent information as if it had been part of the original event, whether or not their retention of the original event is impaired depends on the timing of the postevent information.

Because infants, like the rest of us, usually provide little or no overt indication of what they only passively observe in the course of their daily commerce with the environment, and because memory tokens can be retrieved and new ones can be formed as a consequence of such passive observation, the nature and contents of an individual's changing knowledge base are likely to be underestimated.

\section{REFERENCES}

Amabile, T. A., \& Rovee-Collier, C. (1991). Contextual variation and memory retrieval at six months. Child Development, 62, 1155-1166.

Bekerian, D. A., \& Bowers, J. M. (1983). Eyewitness testimony: Were we misled? Joumal of Experimental Psychology: Learning, Memory, \& Cognition, 9, 139-145.

BELLI, R. F. (1989). Influences of misleading postevent information: Misinformation interference and acceptance. Joumal of Experimental Psychology: General, 118, 72-85.

Belli, R. F., Windschitl, P. D., McCarthy, T. T., \& Winfrey, S. E. (1992). Detecting memory impairment with a modified test procedure: Manipulating retention interval with centrally presented event items. Joumal of Experimental Psychology: Learning, Memory, \& Cognition, 18, 356-367.

Boller, K. \& Rovee-Collier, C. (1991, June). Recoding infants'memories. Paper presented at the meeting of the American Psychological Society, Washington, DC.

Boller, K. , Rovee-Collier, C. (1992). Contextual coding and recoding of infants' memories. Journal of Experimental Child Psychology, 52, 1-23.

CAVE, C. B., \& SQuire, L. R. (1992). Intact and long-lasting repetition priming in amnesia. Journal of Experimental Psychology: Learning, Memory, \& Cognition, 18, 509-520.

Ceci, S. J., Ross, D. F., \& Toglia, M. P. (1987). Age differences in suggestibility: Narrowing the uncertainties. In S. J. Ceci, M. P. Toglia, \& D. F. Ross (Eds.), Children's eyewitness testimony (pp. 79-91). New York: Springer-Verlag.

Ceci, S. J., Toglia, M. P., \& Ross, D. F. (1987) (Eds.). Children's eyewitness testimony. New York: Springer-Verlag.

Chandler, C. C. (1991). How memory for an event is influenced by related events: Interference in modified recognition tests. Journal of Experimental Psychology: Learning, Memory, \& Cognition, 17, 115-125.

Christiaansen, R. E., \& Ochalek, K. (1983). Editing misleading information from memory: Evidence for the coexistence of original and post-event information. Memory \& Cognition, 11, 467-475.

DORIs, J. L. (1991) (Ed.). The suggestibility of children's recollections. Washington, DC: American Psychological Association.

Fagen, J. W., Morrongiello, B. A., Rovee-Collier, C., \& Gekoski, M. J. (1984). Expectancies and memory retrieval in 3-month-old infants. Child Development, 54, 394-403.

Fagen, J. W., Rovee-Collier, C. (1983). Memory retrieval: A timelocked process in infancy. Science, 222, 1349-1351.

GoODMAN, G. S. , \& AMAN, C. (1990). Children's use of anatomically detailed dolls to recount an event. Child Development, 61, 1859-1871. 
Greco, C., Hayne, H., \& Rovee-Collier, C. (1990). Roles of function, reminding, and variability in categorization by 3 -month-old infants. Journal of Experimental Psychology: Human Learning \& Memory, 16, 617-633.

Haist, F., Musen, G., Squire, L. R. (1991). Intact priming of words and nonwords in amnesia. Psychobiology, 19, 275-285.

Hasher, L. ZaCks, R. T. (1979). Automatic and effortful processes in memory. Journal af Experimental Psychology: General, 108 , 356-388.

Hayne, H., Greco, C., Earley, L., Griesler, P., \& RoveeCollier, C. (1986). Ontogeny of early event memory: II. Encoding and retrieval by 2- and 3-month-olds. Infant Behavior \& Development, 9, 441-460.

Hayne, H., Rovee-Collier, C., \& Perris, E. (1987). Categorization and memory retrieval by three-month-olds. Child Development, $\mathbf{5 8}$, $750-767$.

Jenkins, J. G., \& Dallenbach, K. M. (1924). Oblivescence during sleep and waking. American Journal of Psychology, 35, 605-612.

Kahneman, D., \& Treisman, A. (1984). Changing views of attention and automaticity. In R. Parasuraman \& D. R. Davies (Eds.), Varieties of attention (pp. 29-61). New York: Academic.

Kahneman, D., Treisman, A., \&ibbs, B. (1992). The reviewing of object files: Object-specific integration of information. Cognitive Psychology, 24, 175-219.

LEWIS, D. J. (1979). Psychobiology of active and inactive memory. Psychological Bulletin, 86, 1054-1083.

LinDSAY, D. S. (1990). Misleading suggestions can impair eyewitnesses' ability to remember event details. Journal of Experimental Psychology: Learning, Memory, \& Cognition, 16, 1077-1083.

LofTUs, E. F. (1975). Leading questions and the eyewitness report. Cognitive Psychology, 7, 560-572.

LofTus, E. F. (1979). Eyewitness testimony. Boston: Harvard University Press.

Lofrus, E. F. (1981). Mentalmorphosis: Alteration in memory produced by the bonding of new information to old. In J. Long \& A. Baddeley (Eds.), Attention and performance IX (pp. 417-437). Hillsdale, $\mathrm{NJ}$ : Erlbaum.

Lofrus, E. F., \& Hoffman, H. G. (1989). Misinformation and memory: The creation of new memories. Journal of Experimental Psy chology: General, 118, 100-104.

LofTus, E. F., \& LofTus, G. R. (1980). On the permanence of stored information in the brain. American Psychologist, 35, 409-420.

Loftus, E. F., Miller, D. G., \& Burns, H. J. (1978). Semantic integration of verbal information into a visual memory. Journal of $E_{x}$ perimental Psychology: Human Learning \& Memory, 4, 19-31.

Loftus, E. F., Schooler, J. W., \& WagenaAr, W. A. (1985). The fate of memory: Comment on McCloskey and Zaragoza. Joumal of Experimental Psychology: General, 105, 375-380.

McCloskey, M., \& ZARAGozA, M. (1985). Misleading postevent information and memory for events: Arguments and evidence against memory impairment hypotheses. Journal of Experimental Psychology: General, 114, 381-387.

McGaugh, J. L., \& Dawson, R. G. (1971). Modification of memory storage processes. In W. K. Honig \& P. H. R. James (Eds.), Animal memory (pp. 215-242). New York: Academic.

MCGEOCH, J. A. (1942). The psychology of human learning. New York: Longmans, Green.
Murnane, K., Shiffrin, R. M. (1991). Interference and the representation of events in memory. Journal of Experimental Psychology: Leaming, Memory, \& Cognition, 17, 855-874.

Musen, G., \& SQuire, L. R. (1992). Nonverbal priming in amnesia. Memory \& Cognition, 20, 44l-448.

Musen, G., \& Treisman, A. (1990). Implicit and explicit memory for visual patterns. Journal of Experimental Psychology: Learning, Memory, \& Cognition, 16, 127-137.

RoveE, C. K, \& RoveE, D. T. (1969). Conjugate reinforcement of infant exploratory behavior. Journal of Experimental Child Psychology, 8, 33-39.

Rovee-Collier, C., \& DuFault, D. (1991). Multiple contexts and memory retrieval at three months. Developmental Psychobiology, 24, 39-49.

Rovee-Collier, C., Greco-Vigorito, C., \& Hayne, H. (in press). The time window hypothesis: Implications for categorization and memory modification. Infant Behavior \& Development.

ROVEE-COLlIER, C., \& HAYNE, H. (1987). Reactivation of infant memory: Implications for cognitive development. In H. W. Reese (Ed.), Advances in child development and behavior (Vol. 20, pp. 185-238). New York: Academic.

Rovee-Collier, C., Patterson, J., \& Hayne, H. (1985). Specificity in the reactivation of infant memory. Developmental Psychobiology, $18,559-574$.

Rovee-Collier, C., Sullivan, M. W., Enright, M., lucas, D., \& FAGEN, J. W. (1980). Reactivation of infant memory. Science, 208, 1159-1161.

Shields, P. J., \& Rovee-Collier, C. (1992). Long-term memory for context-specific category information at 6 months. Child Development, 63, 245-259.

SмIтн, N. (1968). Effects of interpolated learning on the retention of an escape response in rats as a function of age. Journal of Comparative and Physiological Psychology, 65, 422-426.

SQuire, L. R., DAVIS, H. P. (1981). The pharmacology of memory: A neurobiological perspective. Annual Review of Pharmacology \& Toxicology, 21, 323-356.

Sullivan, M. W. (1982). Reactivation: Priming forgotten memories in human infants. Child Development, 57, 100-104.

Treisman, A. (1992). Perceiving and re-perceiving objects. American Psychologist, 47, 862-875.

TVersky, B., Tuchin, M. (1989). A reconciliation of the evidence on eyewitness testimony: Comments on McCloskey and Zaragoza (1985). Journal of Experimental Psychology: General, 118, 86-91.

Weinberg, H. I., Wadsworth, J., \& BAron, R. S. (1983). Demand and the impact of leading questions on eyewitness testimony. Memory \& Cognition, 11, 101-104.

Zaragoza, M. S. (1991). Preschool children's susceptibility to memory impairment. In J. L. Doris (Ed.), The suggestibility of children's recollections (pp. 27-39). Washington, DC: American Psychological Association.

Zaragoza, M. S., \& Koshmider, J. W., III. (1989). Misled subjects may know more than their performance implies. Journal of Experimental Psychology: Learning, Memory, \& Cognition, 15, 246-255.

Zaragoza, M. S., McCloskey, M., \& JAMIS, M. (1987). Misleading information and recall of the original event: Further evidence against the memory impairment hypothesis. Journal of Experimental Psychology: Learning, Memory, \& Cognition, 13, 36-44.

(Manuscript received October 7, 1991;

revision accepted for publication August 26, 1992.) 